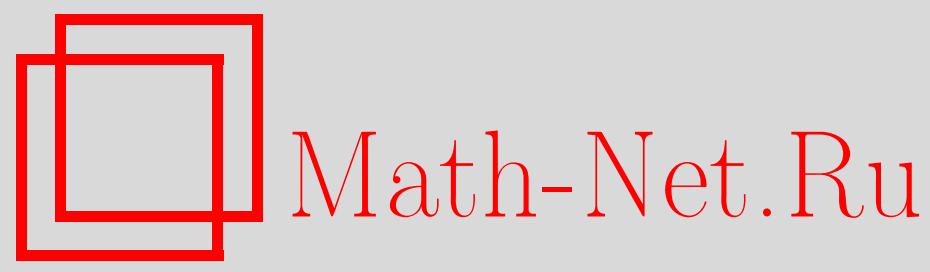

Э. Д. Шваб, Интервалы Ловера и функция Мёбиуса категории Мёбиуса, Дискрет. матем., 2012, том 24, выпуск 4, 4755

DOI: https://doi.org/10.4213/dm1209

Использование Общероссийского математического портала Math-Net.Ru подразумевает, что вы прочитали и согласны с пользовательским соглашением http://www . mathnet.ru/rus/agreement

Параметры загрузки:

IP : 18.207 .199 .55

26 апреля 2023 г., 08:10:46

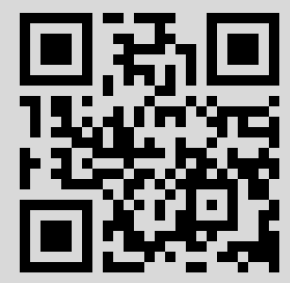




\title{
Интервалы Ловера и функция Мёбиуса категории Мёбиуса
}

\author{
() 2012 г. Э. Д. Шваб
}

\begin{abstract}
Интервалы Ловера категорий Мёбиуса суть конечные однонаправленные категории. В статье изучается простая связь между функцией Мёбиуса категории Мёбиуса и функциями Мёбиуса ее интервалов Ловера. При этом учитывается, что большой класс категорий Мёбиуса возникает как остовы категорий деления (которые являются сократимыми справа с расслоенным копроизведением). Вообще говоря, вычисление функции Мёбиуса для категории Мёбиуса трудоемко. В нашем случае оно становится рутинной операцией на конечных решетках. В качестве иллюстрации, результаты этой статьи использованы для вычисления функции Мёбиуса собственной категории Мёбиуса.
\end{abstract}

\section{1. Введение}

Теория Рота функций Мёбиуса составляет важную часть комбинаторики. Обращения Мёбиуса находят большое число применений в различных областях математики. Функция Мёбиуса $\mu_{P}$ упорядоченного множества $(P, \leqslant)$ полностью определяет обратное исчисление Мёбиуса на $P$. Методы вычисления функции Мёбиуса на некоторых частичноупорядоченных множествах входят в базовую часть этого исчисления. Категории Мёбиуса в смысле Леруа [4] (см. также [1]) были введены для введения обращений Мёбиуса для категорий. Это обобщает теорию Рота функций Мёбиуса для частично-упорядоченных множеств $(P, \leqslant)$. Это обобщение для категорий может быть использовано в различных областях:

(a) решение задач перечисления [6];

(б) теория Эйлеровых характеристик категории [3];

(в) теория комбинаторных обратных моноидов [8];

(г) теория алгебр Хопфа и алгебр инциденций.

Как и в случае теории Рота частично-упорядоченных множеств, в применениях инверсий Мёбиуса в категориях необходимо знать значения функции Мёбиуса на множестве всех морфизмов. В настоящей статье мы приводим ряд понятий и свойств, включая интервалы Ловера, которые облегчают эти вычисления. 


\section{2. Категории Мёбиуса}

Пусть $C$ есть малая категория и $f: X \rightarrow Y$ есть морфизм в $C$. Если $f=g h$ в $C$, мы говорим, что $g$ и $h$ являются множителями $f$ и $g h$ есть факторизация $f$. Обозначим через $\langle f\rangle$ множество всех факторизаций $f$. Назовем малую категорию $C$ декомпозиционноконечной, если множество $\langle f\rangle$ конечно для любого морфизма $f$ малой категории $C$. Комплекснозначная функция $\xi$, определенная на множестве Mor $C$ всех морфизмов $C$ называется функцией инцидентности $C$. Множество $A(C)$ всех функций инцидентности декомпозиционно-конечной категории $C$ с обычной структурой векторного пространства и умножением, заданном конволюцией

$$
(\xi * \eta)(f)=\sum_{g h \in\langle f\rangle} \xi(g) \eta(h)
$$

является $\mathbf{C}$-алгеброй, называемой алгеброй инцидентности $C$. Функция инцидентности $\delta$, определяемая следующим образом:

$$
\delta(f)= \begin{cases}1, & \text { если } f \text { есть тождественный морфизм, } \\ 0 & \text { в противном случае }\end{cases}
$$

есть единичный элемент в $A(C)$. Категория Мёбиуса есть декомпозиционно-конечная категория $C$, в которой функция инцидентности $\xi \in A(C)$ имеет конволюционное обращение тогда и только тогда, когда $\xi(f) \neq 0$ для любого тождественного морфизма $f$. Функция Мёбиуса $\mu$ категории Мёбиуса $C$ есть конволюционное обращение дзетафункции $\zeta$, определенной так, что $\zeta(f)=1$ для любой $f \in \operatorname{Mor} C$. Тогда формула обращения Мёбиуса есть не что иное как следующее утверждение:

$$
\xi=\eta * \zeta \Longleftrightarrow \eta=\xi * \mu \text {. }
$$

Декомпозиционно-конечные однонаправленные категории образуют важный класс категорий Мёбиуса. Говорим, что категория $C$ однонаправленна, если выполняются следующие условия:

(i) из того, что $\operatorname{Hom}_{C}(X, Y) \neq 0$ и $\operatorname{Hom}_{C}(Y, X) \neq 0$ следует, что $X=Y$;

(ii) $\operatorname{Hom}_{C}(X, Y)$ есть синглетон для любого объекта $X$,

где $\operatorname{Hom}_{C}(X, Y)$ - множество всех морфизмов в $C$ из $X$ в $Y$. Заметим, что конечная категория есть категория Мёбиуса тогда и только тогда, когда она однонаправленна. В $[1,2,4]$ приведена очень полезная характеризация категорий Мёбиуса. В [2] характеризации категорий Мёбиуса даны в терминах интервалов. Определение интервала категории было введено Ловером в 1986 г. (см. замечание в [2]). Мы будем называть эти интервалы интервалами Ловера.

\section{3. Интервалы Ловера и функция Мёбиуса}

Пусть $f: X \rightarrow Y$ есть морфизм в категории $C$. Интервал Ловера $I(f)$ есть категория, определяемая следующим образом:

- Ob $I(f)=\langle f\rangle$, 
- $\operatorname{Hom}_{I(f)}\left(f_{1} f_{0}, g_{1} g_{0}\right)=\left\{h \in \operatorname{Mor} C \mid h f_{0}=g_{0}\right.$ и $\left.g_{1} h=f_{1}\right\}$

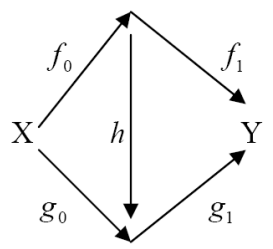

- композиция в $I(f)$ такая же, как и в $C$.

Теорема 1 ([2]). Если $C$ - категория Мёбиуса, то интервал $I(f)$ конечен и мёбиусов для любого морфизма $f$ из $C$. Более того, $C$ мёбиусова тогда и только тогда, когда все интерваль Ловера в С конечны и однонаправленны.

Теорема 2. Если C есть сократимая справа (слева) категория Мёбиуса, то I( $f)$ есть конечное ограниченное частично-упорядоченное множество (то есть, это частичноупорядоченное множество имеет наименьший и наибольший элементы, которые мы обозначим через 0 и 1 соответственно).

Доказательство. Так как все морфизмы категории $C$ являются эпиморфизмами, для любых двух объектов $f_{1} f_{0}, g_{1} g_{0}$ из $I(f)$ множество морфизмов $\operatorname{Hom}_{I(f)}\left(f_{1} f_{0}, g_{1} g_{0}\right)$ представляет собой одноэлементное или пустое множество. Следовательно, $I(f)$ есть частично-упорядоченное множество: $\left(\left(f_{0}, f_{1}\right) \leqslant\left(g_{0} \cdot g_{1}\right)\right.$, если $\operatorname{Hom}_{I(f)}\left(f_{1} f_{0}, g_{1} g_{0}\right)$ - одноэлементное множество. Объект $f 1_{X}$ есть наименьший элемент множества $I(f)$, а $1_{Y} f$ есть наибольший элемент.

Теперь мы несколько изменим направление исследований и перейдем к изучению функций Мёбиуса категорий Мёбиуса. Заметим, что если $C$ есть категория Мёбиуса, то для любого тождественного морфизма $1_{X}$ множество $\left\langle 1_{X}\right\rangle$ одноэлементно. Если $\mu$ есть функция Мёбиуса от $C$, то

$$
\mu\left(1_{X}\right)=\zeta\left(1_{X}\right) \mu\left(1_{X}\right)=(\zeta * \mu)\left(1_{X}\right)=\delta\left(1_{X}\right)=1 .
$$

Если $f: X \rightarrow Y$ есть нетождественный неразложимый морфизм, то есть

$$
\langle f\rangle=\left\{f 1_{X}, 1_{Y} f\right\},
$$

To

$$
\begin{aligned}
\mu(f) & =\zeta\left(1_{Y}\right) \mu(f)=\zeta\left(1_{Y}\right) \mu(f)+1-1 \\
& =\zeta\left(1_{Y}\right) \mu(f)+\zeta f \mu\left(1_{X}\right)-1 \\
& =(\zeta * \mu)(f)-1=\delta(f)-1 \\
& =-1 .
\end{aligned}
$$

Теорема 3. Пусть $\mu$ есть функция Мёбиуса категории Мёбиуса $C$, и пусть $\mu_{I(f)}$ есть функция Мёбиуса интервала Ловера I $(f)$. Тогда

$$
\mu(f)=\mu_{I(f)}(f)
$$

для любого морфизма $f: X \rightarrow Y$ в $C$. (Очевидно, что $f$ есть также морфизм в $I(f)$ из $f_{1_{X}}$ в $\left.1_{Y} f.\right)$ 
Доказательство. Пусть $\xi$ - функция инцидентности категории Мёбиуса $C$, обозначим $\bar{\xi}$ функцию инцидентности $I(f)$, определяемую следующим образом:

$$
\bar{\xi}(h)=\xi(h) \quad \text { для любого морфизма } h \text { из } I(f) .
$$

Теперь пусть $h-$ морфизм из $I(f)$. Если $h=h_{1} h_{0}-$ факторизация $h$ в $I(f)$, то это есть также факторизация $h$ в $C$. Обратно, предположим, что $h=h_{1} h_{0}-$ факторизация $h \in \operatorname{Hom}_{I(f)}\left(f_{1} f_{0}, g_{1} g_{0}\right)$ в $C$. Отсюда следует, что

$$
\left(g_{1} h_{1}\right)\left(h_{0} f_{0}\right)=g_{1} h f_{0}=f_{1} f_{0}=f,
$$

а также, что $h_{0} \in \operatorname{Hom}_{I(f)}\left(f_{1} f_{0},\left(g_{1} h_{1}\right)\left(h_{0} f_{0}\right), h_{1} \in \operatorname{Hom}_{I(f)}\left(\left(g_{1} h_{1}\right)\left(h_{0} f_{0}\right), g_{1} g_{0}\right)\right.$, поэтому $h=h_{1} h_{0}$ есть факторизация $h$ в $I(f)$. Таким образом, $h_{1} h_{2}$ есть факторизация $h$ в $I(f)$ тогда и только тогда, когда она является факторизацией $h$ в $C$. Поэтому для любых двух функций инцидентности $\xi$ и $\eta$ категории $C$ справедливы равенства

$$
\begin{aligned}
(\bar{\xi} * \bar{\eta})(h) & =\sum_{h_{1} h_{0}=h} \bar{\xi}\left(h_{1}\right) \cdot \bar{\eta}\left(h_{0}\right) \\
& =\sum_{h_{1} h_{0}=h} \xi\left(h_{1}\right) \cdot \eta\left(h_{0}\right)=(\xi * \eta)(h) .
\end{aligned}
$$

Следовательно,

$$
\bar{\zeta} * \bar{\mu}=\bar{\mu} * \bar{\zeta}=\delta .
$$

Очевидно, что $\bar{\zeta}$ есть дзета-функция от $I(f)$ и поэтому $\bar{\mu}$ есть функция Мёбиуса $I(f)$.

Теперь для любого морфизма $f: X \rightarrow Y$ в $C f$ есть морфизм в $I(f)$ из $f 1_{X}$ в $1_{Y} f$. Но если $I(f)$ есть ограниченное частично-упорядоченное множество, то $f 1_{X}$ есть наименьший элемент в $I(f)$ (обозначаемый 0) и $1_{Y} f$ есть наибольший элемент (обозначаемый 1 ). Отсюда вытекает следующее утверждение.

Следствие 1. Пусть $\mu$ есть функиия Мёбиуса сократимой справа (слева) категории Мёбиуса $C$ и $\mu_{I(f)}(0,1)$ есть функция Мёбиуса ограниченного частично-упорядоченного множества $I(f)$. Тогда

$$
\mu(f)=\mu_{I(f)}(0,1)
$$

для любого морфизма $f$ в $C$.

Так как Эйлерова характеристика $\chi(P)$ ограниченного конечного частично-упорядоченного множества $P$ есть $\mu_{P}(0,1)$, приходим к следующему утверждению.

Следствие 2. Пусть $\mu$ есть функиия Мёбиуса сократимой справа (слева) категории Мёбиуса С. Тогда

$$
\mu(f)=l_{0}-l_{1}+l_{2}-l_{3}+\ldots
$$

для любого нетождественного морфизма $f$ в $C$, где $l_{i}$ есть число иепочек

$$
0=x_{0}<x_{1}<\ldots<x_{i}=1
$$

в $I(f)$ длинь $і$ между 0 и 1 (считаем $\left.l_{0}=0\right)$. 
Замечание 1. Ряд примеров показывает, что некоторые категории Мёбиуса возникают как остовы категорий деления комбинаторных обратных моноидов (см. $[9,10])$. Все они являются сократимыми справа категориями Мёбиуса. Таким образом, для категорий Мёбиуса комбинаторных обратных моноидов применимы приведенные выше следствия. Более того, все эти категории являются категориями с расслоенным копроизведением (категория деления является сократимой справа категорией с расслоенным копроизведением и квазиинициальным объектом).

Таким образом, верно следующее утверждение.

Теорема 4. Если C есть сократимая справа категория Мёбиуса с расслоенным копроизведением, то любой интервал Ловера в $C$ есть конечная решетка.

Доказательство. Чтобы доказать это утверждение, достаточно показать, что для любого интервала Ловера $I(f)$ и любых двух объектов $f_{0} f_{1}, g_{0} g_{1} \in \mathrm{Ob} I(f)$ найдется наименьшая верхняя грань $f_{1} f_{0} \vee g_{1} g_{0}$. Если $\left[f_{0}, g_{0}, p, q\right]$ есть расслоенное копроизведение в $C$ коугла $\left(f_{0}, d_{0}\right)$, то существует единственный морфизм $u$ в $C$ такой, что $u p=f_{1}$ и $u q=g_{1}$.

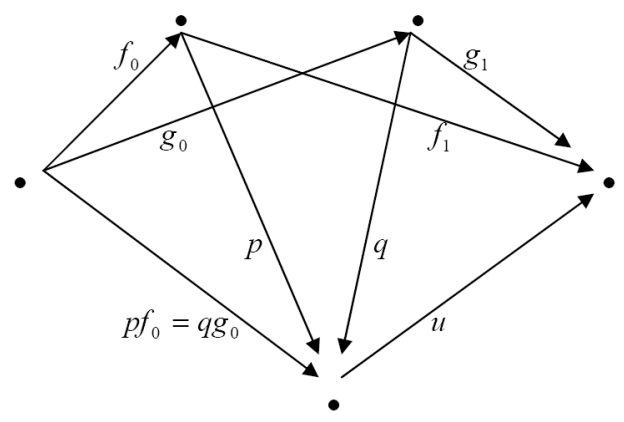

Следовательно, $u\left(p f_{0}\right)=u\left(q g_{0}\right) \in \mathrm{Ob} I(f)$ и $f_{0} f_{1}, g_{0} g_{1} \leqslant u\left(p f_{0}\right)=u\left(q g_{0}\right)$ в $I(f)$. Теперь, если $v w \in \mathrm{Ob} I(f)$ таковы, что $f_{0} f_{1}, g_{0} g_{1} \leqslant v w$, то есть $r f_{0}=w, v r=f_{1}$, $s g_{0}=w$ и $v s=g_{1}$ для некоторых морфизмов $r$ и $s$ в $C$, то $r f_{0}=s g_{0}$. Отсюда следует, что существует единственный морфизм $t$ в $C$ такой, что $t p=r$ и $t q=s$.

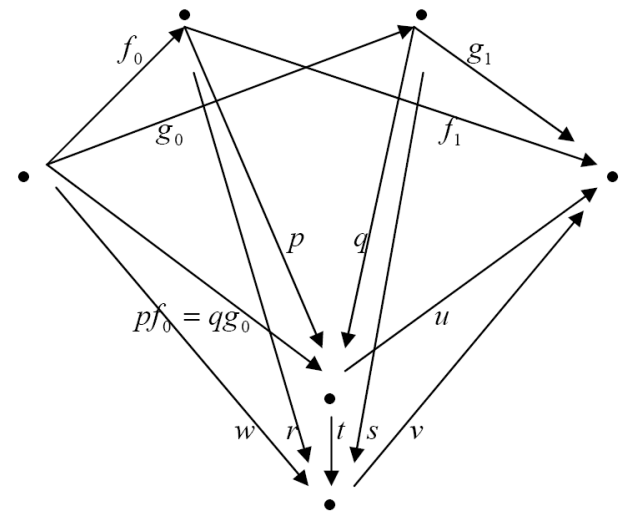

Поэтому равенства

$$
t\left(p f_{0}\right)=(t p) f_{0}=r f_{0}=w, \quad v t q=v s=g_{1}=u q
$$


влекут равенство

$$
v t=u,
$$

и поэтому

$$
u\left(p f_{0}\right)=u\left(q g_{0}\right) \leqslant v w
$$

в $I(f)$. Следовательно, наименьшая верхняя грань для $f_{1} f_{0}, g_{1} g_{0} \in \mathrm{Ob} I(f)$ есть $u\left(h f_{0}\right)=$ $u\left(q f_{0}\right)$.

\section{4. Пример}

Приведем простой пример, иллюстрирующий использование полученных результатов для нахождения функций Мёбиуса категории Мёбиуса. Наибольший интерес в этом примере представляет сам факт, состоящий в том, что в нем представлена собственная категория Мёбиуса (то есть, эта категория - не моноид и она не однонаправленная) и все ее интервалы Ловера являются конечными вполне упорядоченными множествами.

Пусть $Z_{m}=\{\overline{0}, \overline{1}, \ldots, \overline{m-1}\}-$ множество классов вычетов по модулю $m, m \geqslant 2$, и $\mathbf{Z}_{+}$ - множество неотрицательных целых чисел. Рассмотрим категорию $C_{m}$, определенную следующим образом:

$$
\begin{aligned}
\operatorname{Ob} C_{m} & =Z_{m} ; \\
\operatorname{Hom}_{C_{m}}(\bar{a}, \bar{b}) & =\left\{(x, \bar{a}) \in Z_{m} \mid x>a, x \equiv b \quad(\bmod m)\right\} ;
\end{aligned}
$$

если $(x, \bar{a}) \in \operatorname{Hom}_{C_{m}}(a, b)$ и $(y, \bar{b}) \in \operatorname{Hom}_{C_{m}}(\bar{b}, \bar{c})$, то композиция $(y, \bar{b}) \cdot(x, \bar{a})$ задается равенством

$$
(y, \bar{b}) \cdot(x, \bar{a})=(y-b+x, \bar{a}) .
$$

Ясно, что

$$
y-b+x \geqslant a .
$$

Так как

$$
y \equiv c \quad(\bmod v), \quad x \equiv b \quad(\bmod m),
$$

справедливо сравнение

$$
y-b+x \equiv c-b+b \quad(\bmod m),
$$

то есть

$$
y-b+x \equiv c \quad(\bmod m)
$$

Морфизм $(a, \bar{a})$ есть тождественный морфизм из $\bar{a}$ в $\bar{a}$. Очевидно, что категория $C_{m}$ сократима справа и слева. Теперь, если $(a+k, \bar{a})$, где $k \geqslant 0$, есть морфизм из $\bar{a}$ в $\bar{c}$ и

$$
(a+k, \bar{a})=(y, \bar{b}) \cdot(a+i, \bar{a})
$$


есть факторизация $(a+k, \bar{a})$, то

$$
\begin{aligned}
i \geqslant 0, & a+i & \equiv b & (\bmod m), \\
y \geqslant b, & y & \equiv c & (\bmod m),
\end{aligned}
$$

и

$$
y-b+a+i=a+k,
$$

то есть

$$
y=b+k-i \text {. }
$$

Отсюда следует, что для любого $i, 0 \leqslant i \leqslant k$, морфизм $(a+k, \bar{a})$ имеет единственную факторизацию (так как $C_{m}$ сократима) вида

$$
(a+k, \bar{a})=(b+k-i, \bar{b}) \cdot(a+i, \bar{a}),
$$

где

$$
0 \leqslant b \leqslant m-1, \quad b \equiv a+i \quad(\bmod m) .
$$

Отсюда следует, что множество объектов в интервале Ловера $I(a+k, \bar{a})$ имеет следующий вид:

$$
\begin{aligned}
& \mathrm{Ob} I(a+k, \bar{a})=\left\{\left(b_{0}+k, \bar{b}_{0}\right)\right.(a, \bar{a}),\left(b_{1}+k-1, \bar{b}_{1}\right) \cdot(a+1, \bar{a}), \\
& \ldots,\left(b_{i}+k-i, \bar{b}_{i}\right) \cdot(a+i, \bar{a}), \ldots,\left(b_{k}, \bar{b}_{k}\right) \cdot(a+k, \bar{a}),
\end{aligned}
$$

где

$$
0 \leqslant b_{i} \leqslant m-1, \quad b_{i} \equiv a+i \quad(\bmod m), \quad 0 \leqslant i \leqslant k .
$$

Пусть теперь $\left(z, \bar{b}_{i}\right)$ есть морфизм в $I(a+k, \bar{a})$ из $\left.\left(b_{i}+k-i, \bar{b}_{i}\right) \cdot(a+i, \bar{a})\right\}$ в $\left(b_{j}+k-j, \bar{b}_{j}\right) \cdot(a+j, \bar{a})$.

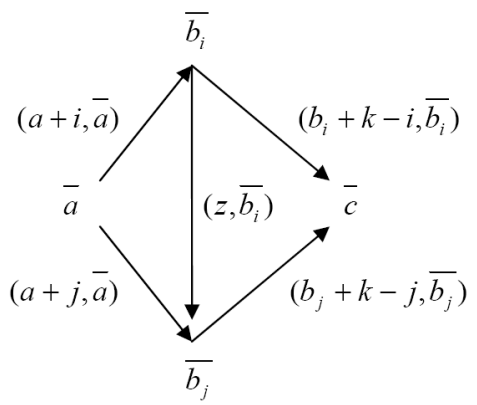

Таким образом, $\left(z, \bar{b}_{i}\right)$ есть морфизм в $C_{m}$ из $\bar{b}_{i}$ в $\bar{b}_{j}$, и поэтому

(1) $z \geqslant b_{i}$

(2) $z \equiv b_{j}(\bmod m)$,

(3) $\left(z, \bar{b}_{i}\right) \cdot(a+i, \bar{a})=(a+j, \bar{a})$ и $\left(b_{j}+k-j, \bar{b}_{j}\right) \cdot\left(z, \bar{b}_{i}\right)=\left(b_{i}+k-i, \bar{b}_{i}\right)$. 
Из (3) следует, что

$$
z=j-i+b_{i}
$$

Так как

$$
a+i \equiv b_{i} \quad(\bmod m), \quad a+j \equiv b_{j} \quad(\bmod m),
$$

верно равенство

$$
j-i+b_{i}=b_{j}
$$

то есть справедливо (2), но (1) справедливо только если $j \geqslant i$. Таким образом, $\operatorname{Hom}_{I(a+k, \bar{a})}\left(\left(b_{i}+k-i, \bar{b}_{i}\right) \cdot(a+i, \bar{a}),\left(b_{j}+k-j, \bar{b}_{j}\right) \cdot(a+j, \bar{a})\right)$ непусто, то есть это есть синглетон, поскольку $C_{m}$ сократимо, если и только если $i \leqslant j(0 \leqslant i, j \leqslant k)$. Следовательно,

(i) все интервалы Ловера в $C_{m}$ конечны и однонаправленны; таким образом, $C_{m}$ есть категория Мёбиуса;

(ii) каждый интервал Ловера $I(a+k, \bar{a})$ категории $C_{m}$ есть цепочка:

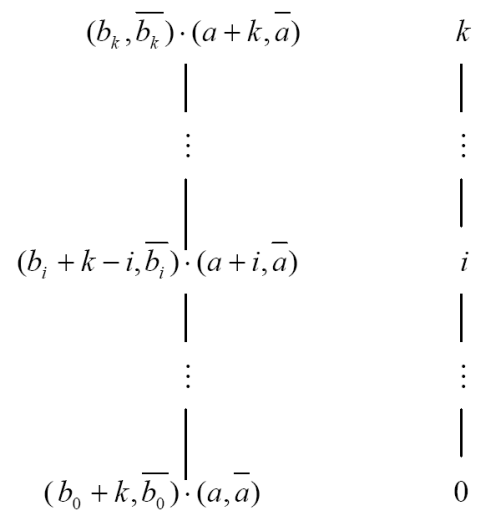

Таким образом, функция Мёбиуса $\mu$ категории Мёбиуса $C_{m}$ задается следующим образом:

$$
\mu(x, \bar{a})= \begin{cases}1, & \text { если } x=a, \\ -1, & \text { если } x=a+1, \\ 0, & \text { если } x \geqslant a+2\end{cases}
$$

для любого морфизма $(x, \bar{a})$ из $C_{m}$.

\section{Список литературы}

1. Content M., Lemay F., Leroux P., Catégories de Möbius et fonctorialités: un cadre général pour l'inversion de Möbius. J. Comb. Theory (1980) A28, №2, 169-190.

2. Lawvere F. W., Menni M., The Hopf algebra of Möbius intervals. Theory and Appl. of Categories (2010) 24, №10, 221-265. 
3. Leinster T., The Euler characteristic of a category. Doc. Math. (2008) 13, 21-49.

4. Leroux P., Les categories de Möbius. Cahiers Topologie Geom. Differentielle (1975) 16, $280-282$.

5. Leroux P., The isomorphism problem for incidence algebras of Möbius categories. Ill. J. Math. (1982) 26, 52-61.

6. Leroux P., Reduced matrices and $q$-log-concavity properties of $q$-Stirling numbers. J. Comb. Theory (1990) A54, 64-84.

7. Rota G.-C., On the foundations of combinatorial theory. Theory of Möbius functions. Z. Wahrscheinlichkeitstheorie (1964) 2, 340-368.

8. Schwab E. D., Möbius categories as reduced standard division categories of combinatorial inverse monoids. Semigroup Forum (2004) 69, 30-40.

9. Schwab E. D., The Möbius category of some combinatorial inverse semigroups. Semigroup Forum (2004) 69, 41-50.

10. Schwab E. D., On incidence algebras of combinatorial inverse monoids. Comm. Algebra (2010) 38, 1779-1789.

11. Stanley R. P., Enumerative combinatorics, I. Wadsworth \& Brooks, Monterey, Calif., 1986.

Статья поступила 20.08.2011.

Переработанный вариант поступил 10.11.2012. 